\title{
Impaired Sociability of the Balb/c Mouse, an Animal Model of Autism Spectrum Disorders, is Attenuated by NMDA Receptor Agonist Interventions: Clinical Implications
}

\author{
Stephen I. Deutsch, Jessica A. Burket, \\ Maria R. Urbano, Amy L. Herndon and Erin E. Winebarger \\ Eastern Virginia Medical School, Norfolk VA \\ United States of America
}

\section{Introduction}

Impaired sociability is a domain of psychopathology that contributes significantly to the functional disability and poor quality of life of persons with Autism Spectrum Disorders (ASDs) (Brodkin, 2007; Crawley, 2004, 2007; Deutsch et al., 2011). Although cognitive functioning may influence the expression of impaired sociability, the relationship between cognitive deficits and impaired sociability is not a linear one; thus, for example, persons with ASDs whose IQs are in the near-normal to above normal range may manifest profound deficits of sociability that are similar to those shown by persons with ASDs and intellectual disability (Noterdaeme et al., 2010). Currently, there are no approved medications whose primary target is the domain of impaired sociability.

The N-methyl-D-aspartic acid (NMDA) receptor is an example of a glutamate-gated ion channel that is comprised of four (or possibly five) constituent polypeptide subunits; it is located both pre- and postsynaptically (Millian, 2005). Each constituent polypeptide is an integral membrane protein that has external, transmembranous and internal domains. The constituent polypeptides align themselves within the lipid bilayer of the membrane to create a potential channel, whose opening depends on the membrane potential and the binding of L-glutamate and glycine. The duration and frequency of channel opening, which results in calcium ion conductance and membrane depolarization, are highly regulated. The properties of this ligand-gated ion channel receptor are influenced genetically by the combinatorial diversity of the constituent polypeptide subunits (i.e., expression and membrane insertion of specific subunits and their splice variants can affect channel properties); allosteric modulatory ligands (e.g., neurosteroids) (Deutsch et al., 1992); the extent to which the receptor is glycosylated and nitrosylated; and the extent to which the internal domain of receptor polypeptide subunits are phosphorylated (Marino \& Conn, 2002). The phosphorylation state of the internal domain is influenced by cross-talk between specific signaling pathways and the NMDA receptor; for example, stimulation of metabotropic glutamate receptors co-localized with NMDA receptors on the cell surface can influence the extent of phosphorylation (Conn et al., 2009a; Marino \& Conn, 2002). When the 
membrane is at its resting potential, a hydrated magnesium ion occupies a binding site within the hydrophobic channel domain of the NMDA receptor; this magnesium ion blockade is removed as the receptor depolarizes, which may occur as a result of stimulating non-NMDA excitatory glutamate-gated ion channel receptors co-localized with NMDA receptors on the membrane surface (Chen et al., 1992). Once the magnesium ion blockade is removed with "depolarization" of the membrane, L-glutamate and glycine interact allosterically with each other to increase the likelihood that the channel will assume an open configuration that permits calcium ion conductance (Marino \& Conn, 2002). Presynaptically, the opening of the NMDA receptor may be involved in calcium ion-dependent release of transmitters; channel opening results in the transient increase of the intraneuronal calcium ion concentration (Marino \& Conn, 2002). Postsynaptically, NMDA receptors located on the surface of GABAergic inhibitory neurons contribute to regulation of the inhibitory tone of the brain (Benes, 2010). Functionally, because L-glutamate is one of the most abundant neurotransmitters in the brain, NMDA receptors are involved in many neural circuits; for example, NMDA receptors are necessary for the induction of long-term potentiation in the hippocampus and have a major role in spatial memory performance referable to the hippocampus, among many other functions (Benes, 2010).

Targeted mutations in mice that affect expression of the NR1 subunit of the NMDA receptor and its affinity for glycine, the obligatory co-agonist that binds to the NR1 subunit and contributes to regulation of channel opening, have been created (Halene et al., 2009; Labrie et al., 2008). Interestingly, in a standard sociability paradigm, these genetically-engineered mice with diminished expression of the NR1 subunit or five-fold diminished affinity of the NR1 subunit for glycine show deficits in sociability ((Halene et al., 2009; Labrie et al., 2008). Thus, altered NMDA receptor-mediated neurotransmission in mice is associated with impaired sociability (e.g., these mutant mice make fewer social approaches and spend less time in the vicinity of an enclosed or freely-moving socially-salient stimulus mouse).

The genetically inbred Balb/c mouse strain displays behavioral hypersensitivity to MK-801 (dizocilpine), a noncompetitive NMDA receptor antagonist (Billingslea et al., 2003; Burket et al., 2010a; Deutsch et al., 1997, 1998). Thus, MK-801 is more potent at antagonizing electrically-precipitated seizures in Balb/c mice than a variety of other genetically-inbred and outbred mouse strains (Billingslea et al., 2003; Deutsch et al., 1998). Additionally, Balb/c mice are more sensitive than other reference mouse strains to the elicitation of irregular episodes of intense jumping behavior, which has been referred to as "popping" (Billingslea et al., 2003; Deutsch et al., 1997). Further, relative to the outbred Swiss-Webster mouse strain, Balb/c mice engaged in more intense MK-801-elicited circling behavior (Burket et al., 2010a). The heightened behavioral sensitivity of the Balb/c mouse to MK-801 does not appear to be due to a "defect" in the receptor itself because a Western Blotting experiment revealed no differences in the immunoreactive content of six of the eight identified splice variant isoforms of the NR1 subunit, and NR2A and NR2B subunits in the hippocampus and cerebral cortex of Balb/c and NIH Swiss mice (Perera et al., 2008). Thus, the altered endogenous tone of NMDA receptor-mediated neurotransmission in the Balb/c mouse strain may be due to factors "upstream" or "downstream" of the receptor itself. In any event, the Balb/c mouse strain, like the targeted genetic mutant mouse strains described above, shows quantitative deficits of sociability in a standard mouse paradigm (Burket et al, 2010b; Brodkin, 2007; Moy et al., 2007; Sankoorikal et al., 2006). These deficits of sociability are observable when Balb/c mice are in the presence of a salient social stimulus mouse that is either enclosed or allowed to interact freely with a Balb/c mouse (Burket et al., 2010b; 
Deutsch et al., 2011; Jacome et al., 2011a,b, in press). Specifically, Balb/c mice make fewer discrete episodes of social approach and spend less time exploring (sniffing) and in the vicinity of an enclosed and freely-moving social stimulus mouse (Jacome et al., 2011; Brodkin, 2007). Quite remarkably, D-cycloserine, a partial glycine agonist for the obligatory co-agonist binding site, and D-serine, a full agonist for this site that may also be the endogenous ligand, were able to attenuate the severity of the Balb/c mouse's deficits of sociability (Deutsch et al., 2011; Jacome et al., 2011a,b). These effects of NMDA receptor agonist interventions may be due to direct effects on the sociability deficit itself, as opposed to nonspecific effects resulting from increased locomotor activity in general. These preclinical results are also consistent with a pilot clinical trial of D-cycloserine administration to 10 children with ASDs; in this pilot investigation, D-cycloserine improved scores on the social withdrawal subscale of the Aberrant Behavior Checklist (Posey et al., 2004). Importantly, persons with ASDs may have deficient central inhibitory tone, consistent with the high prevalence of co-morbid seizures, which would benefit from an NMDA receptor agonist intervention.

In summary, the Balb/c mouse has emerged as a genetic mouse model of impaired sociability that is useful for screening targeted NMDA receptor agonist interventions, which may be candidates for development as medications to treat impaired sociability, a "neglected" domain of psychopathology in persons with ASDs. The topic areas introduced in this overview will be considered below in greater detail.

\section{Impaired glutamatergic transmission may be a shared pathophysiological mechanism among persons with diverse etiologies of their autism spectrum disorders}

Autism Spectrum Disorders (ASDs) are a heterogeneous group of disorders with respect to their clinical presentations that are highly heritable, as shown in family and twin studies (Toro et al., 2010); however, with the exception of ASDs occurring in the context of genetic disorders due to the effects of a single gene (e.g., tuberous sclerosis), the nature of what is inherited and the mode of genetic transmission are usually not known for most patients (Toro et al., 2010). Moreover, even in cases where ASDs are due to the effects of a single major mutant gene, the steps via which a single mutant gene leads to an autism syndrome are also unclear. In spite of the variability of their clinical presentations, persons with the diagnosis of an ASD manifest symptoms in three operationally-defined domains of psychopathology: "qualitative impairment in social interaction;" "qualitative impairment in communication;" and "restricted repetitive and stereotyped patterns of behavior (American Psychiatric Association 2000)." Persons with Asperger's Disorder, however, may have little or no clinically significant delays in language and cognitive development. Recent genetic investigations suggest that persons with ASDs may have "hotspots" along the genome where microdeletions or duplications of DNA, referred to as Copy Number Variations or CNVs, are more likely to occur (Bremer et al., 2011). CNVs can lead to problems related to "gene dosing effects (i.e., either absent or too much expression of genes that contribute to emergence of ASDs)" or, alternatively, can affect the coiling and higher-order structure of double-stranded DNA, which, in turn, affects transcription (Toro et al., 2010). Another possibility is that a microdeletion can lead to unmasking and unopposed expression of a "recessive" mutant gene product, whose homozygous expression may be ordinarily lethal in utero. Additionally, since the etiologies of ASDs vary but persons with ASDs share 
symptoms in the three discrete domains of autism psychopathology, there is interest in identifying discrete neural circuits that may serve as the substrate for impaired socialemotional cognition and other domains of psychopathology in ASDs, irrespective of etiology, and exploring the efficiency of synaptic transmission between nodes within these neural circuits in affected persons. Thus, imaging studies, such as functional MRI, examine activation of specific nodes, such as amygdala, fusiform gyrus, anterior cingulate cortex, among other brain areas, while subjects with ASDs view projected images of the human face displaying various facial expressions or "affects," such as fear, anger, disdain or happiness (Kleinhans et al., 2011; Ashwin et al., 2007). Moreover, disturbances within circuits may be responsible for the abnormal facial scanning strategies persons with ASDs employ (e.g., spending less time gazing at the eyes relative to typically developing normal subjects). Because pathophysiological abnormalities may represent a common final pathway that many persons with ASDs share in-common, irrespective of etiology, it may be possible to design medication strategies that target inefficient or aberrant synaptic transmission along neural circuits (e.g., the neural circuit necessary for normal social-emotional cognition) that would be beneficial for an etiologically diverse group of persons with ASDs (Table 1).

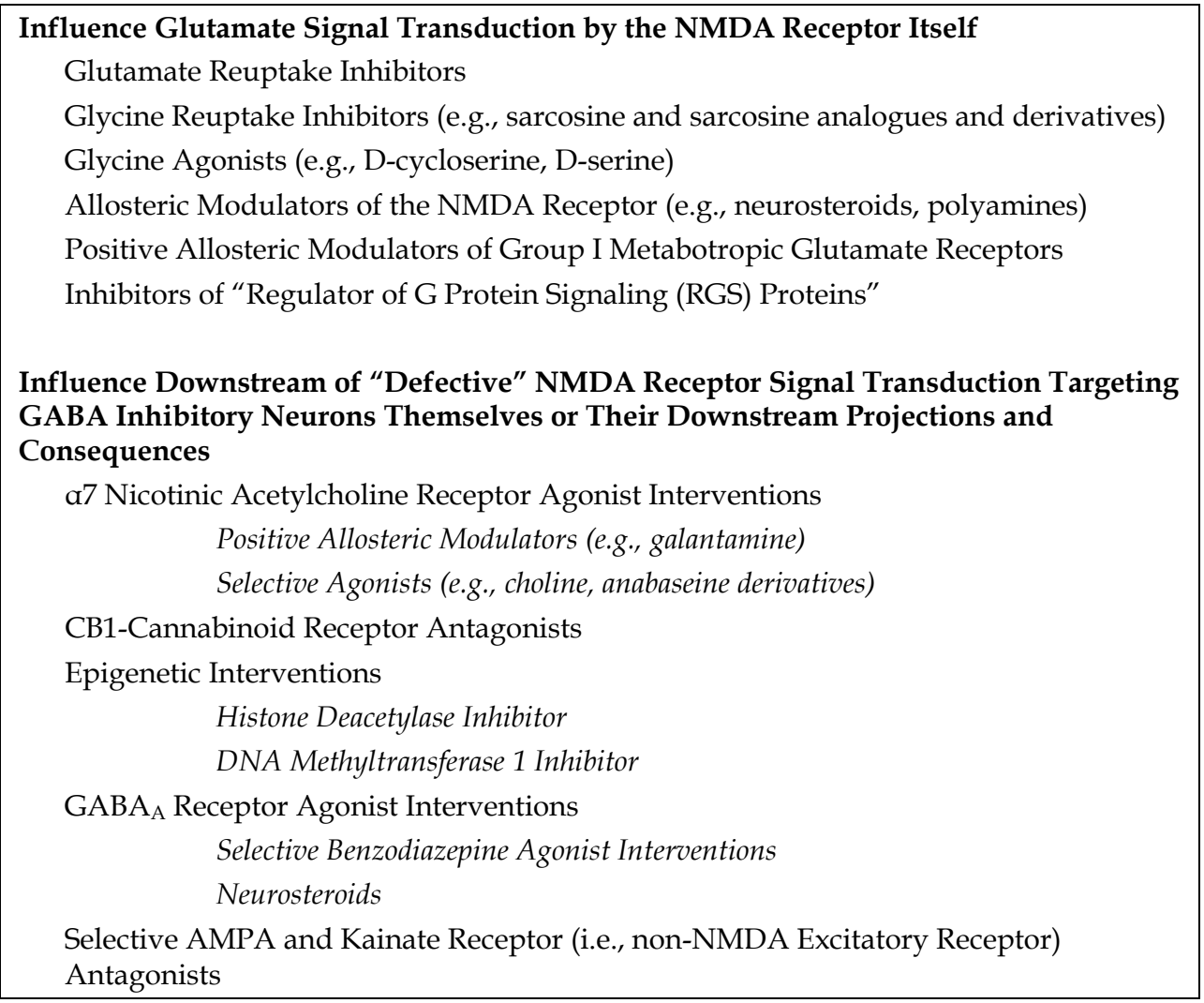

Table 1. Potential Pharmacological Strategies to Manipulate Endogenous Tone of NMDA Receptor-Mediated Neurotransmission 
Conceivably, impairments of sociability manifested by persons with ASDs reflect disturbances within the social-emotional neural circuit (Benes, 2010). Given the quantitative importance of glutamatergic transmission in the brain and recent data suggesting that diminished or defective expression of the N-methyl-D-aspartic acid (NMDA) subtype of ionotropic glutamate receptor is causally-related to deficits of sociability in transgenic mouse strains, the NMDA receptor has emerged as a medication target to improve sociability in persons with ASDs (Halene et al., 2009; Duncan et al., 2004). More specifically, NMDA receptors are involved in regulating presynaptic release of a variety of neurotransmitters, including glutamate itself, and their postsynaptic location on the surface of GABA inhibitory neurons plays a very important role in regulating central inhibitory tone and critical oscillatory rhythms in brain (see below) (Benes, 2010, Deutsch et al., 2010). GABA inhibitory neurons project to assemblies of pyramidal neurons and it is the oscillatory firing of these latter assemblies of neurons that underlie key rhythms in the brain; the power spectra of gamma oscillations are associated with working memory (Benes, 2010; Deutsch et al., 2010). Importantly, pyramidal outflow neurons utilize glutamate as their neurotransmitter.

\section{NMDA receptor - Structure and function}

The NMDA receptor is a highly-regulated ligand-gated ion channel that also has features of voltage-gating; although it is a ligand-gated ion channel receptor, it does not enjoy the close homology and genetic-relatedness that nicotinic acetylcholine and $\mathrm{GABA}_{\mathrm{A}}$ receptors share with each other. However, like other ligand-gated ion channel receptors, the NMDA receptor is an oligomeric protein constructed from four constituent polypeptide subunits, each of which shares a common motif; nicotinic and $\mathrm{GABA}_{\mathrm{A}}$ receptors have five subunits (Millan, 2005). The receptor polypeptide subunits are themselves integral membrane proteins that have an N-terminal extracellular domain, four hydrophobic transmembranous domains (in fact, the third hydrophobic loop does not span the entire width of the lipid bilayer), and an intracytoplasmic loop that has consensus sequences of amino acids that serve as substrates for phosphorylation by specific kinases (Conn et al., 2009a; Marino \& Conn, 2002). Phosphorylation of the intracytoplasmic loop influences the gating and channel properties of the ionophore and often occurs as a result of "cross-talk" with G-protein coupled-receptors (GPCR) located next to or near the NMDA receptor on the surface of the neuron (Conn et al., 2009a; Gregory et al., 2011). The constituent polypeptide subunits align themselves in such a manner that they form a potential pore or channel in the membrane, whose opening is dependent on the binding of glutamate and its obligatory glycine co-agonist. The filtering properties of the channel are such that calcium ions can flux through the open channel along their electrochemical gradient. At the ordinary resting membrane potential, a hydrated magnesium ion is bound within the channel; in order for the channel to become functional, this magnesium ion blockade must be relieved, which occurs as the membrane begins to depolarize. Thus, functional conductance through the NMDA receptor is dependent on membrane depolarization, which may occur through the actions of glutamate stimulating AMPA receptors, another excitatory glutamategated ion channel receptor that may reside in the vicinity of NMDA receptors. The functional properties of NMDA receptors themselves are influenced by the unique combinations of NR1 splice variant isoforms with NR2A and NR2B receptor subunits (Kew \& Kemp, 2005). Functional receptors are dependent on inclusion of the NR1 
subunit or one of its eight common splice variants; the usual combinations comprising functional NMDA receptors involve two obligatory NR1 and either two NR2A or two NR2B subunits (Kew \& Kemp, 2005). Functional NMDA receptors may have two glycine binding sites, whose construction depends on the extracellular domains of NR1 subunits, and two glutamate binding sites (Kew \& Kemp, 2005; Millan, 2005). Also, as noted above, the extent to which the intracytoplasmic domain is phosphorylated, and the local $\mathrm{pH}$ and oxidation potential surrounding the receptor influence the receptor's channel properties (i.e., the likelihood the channel will open and frequency of channel openings in response to the binding of glutamate and its obligatory glycine co-agonist to specific sites in the extracellular domain of the NMDA receptor)(Millan, 2005; Stephenson, 2006). The likelihood that glutamate and glycine will be effective in promoting channel opening is also influenced by levels of neurally-active steroids, which act as allosteric modulators (Deutsch et al., 1992). Neurally-active steroids are derived from peripheral steroid hormones and also are synthesized in situ in the brain by glial cells; the highly-regulated translocation of cholesterol across the mitochondrion is the first step in neurosteroid biosynthesis in the brain. Clearly, glutamate-gated calcium ion flux across the NMDA receptor-associated ionophore is a highly regulated process, subject to genetic influences (i.e., combinatorial diversity or the unique combination of receptor polypeptide subunits and their splice variants that comprise the receptor and influence its channel properties); post-translational modifications (e.g., the extent to which the receptor is phosphorylated and nitrosylated); and circulating levels of endogenous allosteric modulators (e.g., neurosteroids and polyamines, such as spermidine) (Conn et al., 2009a; Deutsch et al., 1992; Kew \& Kemp, 2005, Millan, 2005; Stephenson, 2006).

NMDA receptors have both pre- and postsynaptic localizations on neurons. Presynaptically, NMDA receptors may participate in neurotransmitter release, a process dependent on calcium ions, whereas postsynaptically, their location on GABAergic neurons may contribute to the regulation of inhibitory tone and normal oscillatory activity in brain (Deutsch et al., 2010). Interestingly, seizures occur commonly in persons with autism spectrum disorders, consistent with diminished central inhibitory tone; also, the normal gamma oscillatory rhythm and power spectra of gamma oscillations, an important oscillatory rhythm associated with working memory and higher executive functions, are dependent on functional GABAergic projections to assemblies of pyramidal cell output neurons (Deutsch et al., 2010). Thus, the integrity of circuits necessary for normal cognition and social behavior, whose nodes include cerebral cortex, anterior cingulate gyrus, hippocampus, and amygdala, among other discrete anatomic brain regions, contain NMDA receptors (Benes, 2010). An emerging body of preclinical and clinical data suggests that NMDA receptor hypofunction at the level of the receptor itself or due to abnormalities upstream or downstream of the receptor contributes to both cognitive problems and impaired sociability (Darrah et al., 2008; Deutsch et al., 2011; Halene et al., 2009; Jacome et al., 2011b; Labrie et al., 2008).

The ability of glycine to make glutamate a more efficient neurotransmitter and, thereby, increase the likelihood the channel will transiently assume an open configuration and allow calcium ions to flux freely has aroused interest in the strychnine-insensitive obligatory glycine co-agonist binding site on the NMDA receptor as a target for medication development to address presumptive NMDA receptor hypofunction. The strychnine-insensitive glycine binding site on the NMDA receptor is also referred to as the glycine $_{\mathrm{B}}$ site (Millan, 2005). Interestingly, D-serine may be the naturally-occurring and 
preferred ligand for the glycine binding site on the NMDA receptor; a racemase exists that converts the more commonly-occurring L-form of serine, which is incorporated into protein and extensively involved in intermediary metabolism, to the D-form, which may have a unique localization and function within brain (Jacome et al., 2011b; Long et al., 2006). Exogenously-administered D-serine and other directly acting full agonists may serve as "proofs of concept" to implicate NMDA receptor hypofunction in some aspects of the pathophysiology of autism spectrum disorders, especially in animal models studying the effects of their single dose administration on outcome measures related to sociability and cognition (Jacome et al., 2011b). However, directly-acting full glycine $\mathrm{B}_{\mathrm{B}}$ agonists may have limitations with respect to their development as medication interventions, which would have to be administered chronically in disorders such as autism spectrum disorders, because of concerns about "agonist-induced" receptor desensitization and excitotoxicity (Jacome et al., 2011b). Thus, in terms of viable medication strategies, there is interest in partial glycine agonists (e.g., D-cycloserine), and influencing NMDA receptor channel properties indirectly through "cross-talk" with other receptors (e.g., strategies exploring administration of positive allosteric modulators [PAMs] of type 1 metabotropic glutamate receptors [mGluRs]). Specifically, our laboratory is interested in exploring the effect of PAMs of mGluR5 on the impaired sociability of Balb/c mice, a genetic mouse model of autism spectrum disorders, because mGluR5 stimulation leads to phosphorylation of a specific serine residue within the intracytoplasmic domain of the NR1 subunit (Takagi et al., 2010). In general, as members of class “C" G-protein coupledreceptors, mGluR5 receptors are constitutive dimers that are located postsynaptically in close functional proximity to NMDA receptors. They have a large extracellular 'venus flytrap domain (VFD)' that is the L-glutamate agonist recognition site; binding of L-glutamate leads to the propagation of a conformational change via 'cysteine-rich domains (CRD),' resulting in activation of phospholipase $C \beta$ through its coupling to ligand-binding by a Gq, a specific GTP binding protein (Niswender \& Conn, 2010). A downstream consequence of phospholipase activation includes increased phosphorylation of the NMDA receptor. There are many functional consequences of increasing the phosphorylation status of NMDA receptors, several of which may be relevant to domains of psychopathology present in ASDs. For example, MK-801 can disrupt cognitive flexibility or set-shifting ability; the severity of this disruption can be attenuated by a positive allosteric modulator of mGluR5 receptors (Darrah et al., 2008). Presumably, this positive therapeutic effect reflects the increased phosphorylation state of NMDA receptors. As will be discussed, functional NMDA receptors and "normal" tone of endogenous NMDA receptor-mediated neurotransmission are necessary for "normal" sociability in mice.

\section{NMDA receptor and genetic mouse models of autism spectrum disorders}

The NMDA receptor has emerged as a promising target for medication strategies designed to improve sociability (and cognition) in studies involving both geneticallyengineered and inbred mouse strains, and other rodent species (Blundell et al., 2010; Deutsch et al, 2011; Darrah et al., 2008; Halene et al., 2009; Jacome et al., 2011b; Labrie et al., 2008). Importantly, the NMDA receptor is known to be involved in important aspects of electrophysiology relevant to cognition (e.g., induction of long-term potentiation in the hippocampus) and memory performance (e.g., hippocampally-dependent spatial 
memory). These findings were complemented with relatively recent investigations showing transgenic mice with diminished expression of the NR1 subunit of the NMDA receptor ( 5 to $10 \%$ of normal levels of expression) or five-fold diminished affinity of the receptor for glycine as a result of targeted mutagenesis of the glycine binding domain on the NR1 subunit have quantitative deficits of sociability in a standard paradigm (Halene et al., 2009; Labrie et al., 2008). Perhaps, of even greater significance, a targeted NMDA receptor agonist intervention (i.e., D-cycloserine) attenuated measurable deficits of sociability in "genetically-normal" resident and intruder mice in a standardized social interaction paradigm (McAllister, 1994). Thus, even in the absence of a genetic lesion affecting the NMDA receptor itself, this receptor could serve as a target to improve sociability and cognition. Additionally, encouraging data are emerging from studies with genetic mouse models of autism spectrum disorders, which are neurodevelopmental disorders, that pharmacotherapeutic interventions can improve quantitative deficits of sociability in young adult mice (Deutsch et al., 2011; Jacome et al., 2011a,b; Sankoorikal et al., 2006). Thus, there is reason for optimism with respect to potential for effectiveness of newly-developed medications identified in standard sociability paradigms using transgenic and inbred mouse strains as subjects; unfortunately, autism spectrum disorders are not often recognized in preschool-age children and, as a result, treatment is often delayed, which is why the positive data with young adult mice are so encouraging.

\section{Sociability paradigm}

Our laboratory has adopted a recently established mouse behavioral procedure for the quantitative assessment of sociability (Brodkin, 2007; Burket et al., 2010b; Crawley, 2004, 2007; Deutsch et al., 2011; Jacome et al., 2011b; Moy et al., 2007; Sankoorikal et al., 2006; Silverman et al., 2010). Briefly, in the first session, a test mouse is placed in the middle compartment and allowed to acclimate to the sociability apparatus for 5 minutes. In the second 5-minute session, a stimulus mouse is enclosed in an inverted wire cup in the side designated as the social compartment, and an empty inverted wire cup is placed in the side designated as the nonsocial compartment. The side designated for the location of the enclosed stimulus mouse is randomly assigned in a counterbalanced fashion throughout the experiment. In the third 5-minute session, the stimulus mouse is released from the inverted wire cup, and the test and stimulus mice are allowed to interact freely with each other (Figure 1). All sessions are conducted in dim lighting and videotaped for future viewing and data collection.

The amount of time a test mouse spends in the social and nonsocial compartments and exploring (sniffing) the inverted wire cups is measured in session II. Locomotor activity is measured in all three sessions and is defined as the number of transitions (i.e., number of times all four extremities cross each compartment) the test mouse makes across all three compartments in each 5-min session. Other behavioral measures of the test mouse include additional measures of sociability (i.e., social approach, social avoidance, social pursuit, mounting and anogenital sniffing) and stereotypic behaviors (i.e., rearing, grooming and wall climbing), which are obtained during the 5-minute session of free interaction between test and stimulus mice (Table 2). Importantly, our laboratory showed that these behavioral measures can be reliably rated by independent observers (Jacome et al., in press). 

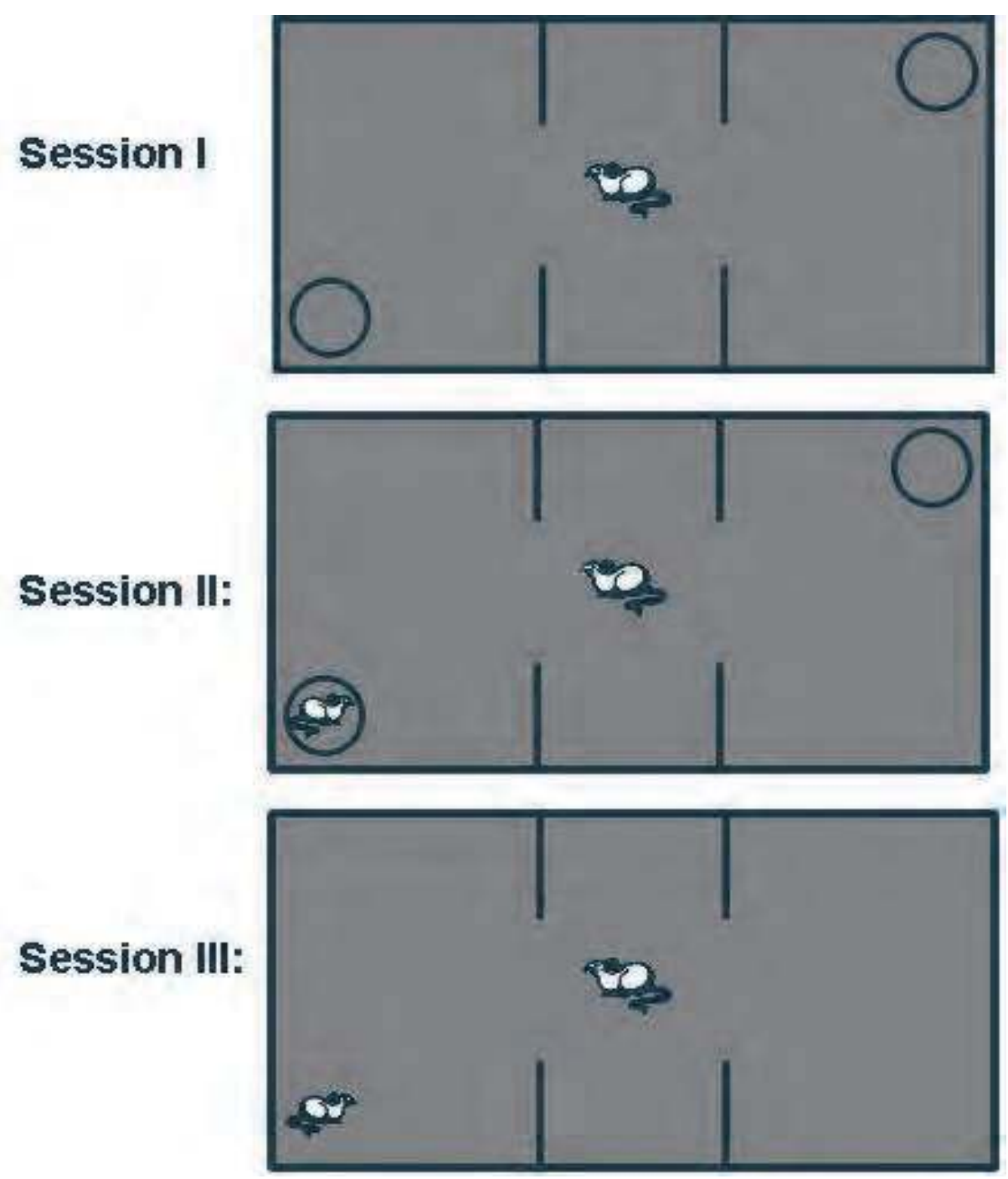

Fig. 1. Sociability paradigm 


\begin{tabular}{|c|c|}
\hline \multicolumn{2}{|c|}{ Measures of Sociability } \\
\hline Social approach & $\begin{array}{l}\text { A social behavior of test and stimulus mice defined as a discrete } \\
\text { episode of their initiation of sniffing within a two-cm vicinity of each } \\
\text { other. }\end{array}$ \\
\hline $\begin{array}{l}\text { Social } \\
\text { avoidance }\end{array}$ & $\begin{array}{l}\text { A nonsocial response of the test mouse defined as a discrete episode of } \\
\text { freezing, withdrawing, or turning its head away while within a two-cm } \\
\text { vicinity of the socially-salient stimulus mouse. }\end{array}$ \\
\hline Social Pursuit & $\begin{array}{l}\text { A measure of the time that the test mouse spends following or chasing } \\
\text { the socially-salient stimulus mouse from initiation of the encounter } \\
\text { until both mice separate by a distance of at least two cm. }\end{array}$ \\
\hline $\begin{array}{l}\text { Anogenital } \\
\text { sniffing }\end{array}$ & $\begin{array}{l}\text { A social behavior displayed by the test mouse defined as a discrete } \\
\text { episode of sniffing the anogenital area of the stimulus mouse within a } \\
\text { two-cm vicinity. }\end{array}$ \\
\hline Mounting & $\begin{array}{l}\text { A social behavior displayed by the test mouse defined as a discrete } \\
\text { episode of placing the front paws on the stimulus mouse. }\end{array}$ \\
\hline \multicolumn{2}{|c|}{ Measures of Stereotypic Behaviors } \\
\hline Rearing & $\begin{array}{l}\text { A stereotypic behavior displayed by the test mouse defined as a } \\
\text { discrete episode of raising forelimbs and standing on hindlimbs. }\end{array}$ \\
\hline Grooming & $\begin{array}{l}\text { A stereotypic behavior displayed by the test mouse defined as a } \\
\text { discrete episode of licking itself. }\end{array}$ \\
\hline Wall climbing & $\begin{array}{l}\text { A stereotypic behavior displayed by the test mouse defined as a } \\
\text { discrete episode of raising forelimbs and placing front paws on walls of } \\
\text { the sociability apparatus. }\end{array}$ \\
\hline
\end{tabular}

Table 2. Measures of Sociability and Stereotypic Behavior

\section{The genetically-inbred balb/c mouse, a mouse strain that is behaviorally- hypersensitive to mk-801 (dizocilpine), a noncompetitive nmda receptor antagonist, shows quantitative deficits of sociability}

Over the past 20 years, our laboratory has been exploring the effects of stress on the abilities of flurazepam, a benzodiazepine agonist, and MK-801 (dizocilpine), a noncompetitive NMDA receptor antagonist, to antagonize electrically-precipitated tonic hindlimb extension in mice (Billingslea et al., 2003; Burket et al., 2009; Deutsch et al., 1990; 1998). These studies showed that 24 hours after mice are forced to swim for up to 10 minutes in cold $\left(6^{\circ} \mathrm{C}\right)$ water, the abilities of flurazepam and MK-801 to antagonize electrically-precipitated seizures are reduced. During the course of these investigations, it 
was observed that the genetically-inbred Balb/c mouse strain showed increased sensitivity to MK-801 administration on several behavioral measures. For example, relative to other inbred and outbred strains, the potency of MK-801 to raise the threshold voltage for elicitation of tonic hindlimb extension was greater in the Balb/c mouse (Billingslea et al., 2003; Deutsch et al., 1998). Similarly, Balb/c mice were more sensitive to the ability of MK-801 to elicit irregular episodes of intense jumping behavior, referred to as "popping," and "circling" behavior than other comparator strains (Billingslea et al., 2003; Burket et al., 2010a; Deutsch et al., 1997). The increased sensitivity to behavioral effects of MK-801 suggested to us that there is something "altered" in the endogenous tone of NMDA receptor-mediated neurotransmission in the Balb/c strain, relative to the other strains that we examined. Surprisingly, when we studied the immunoreactive protein content of six of the eight common splice variant isoforms of the NR1 subunit and NR2A and NR2B subunits in hippocampi and cerebral cortices from Balb/c and an outbred comparator strain, no significant differences were evident (Perera et al., 2008). These data suggested to us that the presumed altered endogenous tone of NMDA receptor-mediated neurotransmission in the Balb/c mouse was not at the level of the NMDA receptor itself (e.g., due to altered expression of the receptor polypeptide subunits), but, perhaps, at synapses upstream or downstream of the location of the receptor.

Because of our interest in the Balb/c mouse, we replicated reports that this mouse strain showed quantitative deficits of sociability in a standard paradigm (Brodkin, 2007; Moy et al., 2007; Sankoorikal et al., 2006), and extended these observations to include a detailed study of its locomotor activity in the presence of an enclosed salient social stimulus mouse and when Balb/c and stimulus mice are allowed to interact freely with each other. These studies showed that the locomotor activity of the Balb/c mouse strain is suppressed in the presence of a social stimulus mouse, which serves as another quantitative measure of impaired sociability (Burket et al., 2010b). The Balb/c mouse makes fewer discrete episodes of social approach, displays more social avoidance, and spends less time exploring and in the vicinity of both an enclosed and freely-behaving social stimulus mouse, than the outbred Swiss Webster comparator strain (Deutsch et al., 2011; Jacome et al., 2011b; submitted manuscript). Moreover, the impaired sociability of the Balb/c mouse affects the behavior of the social stimulus mouse when they are allowed to freely interact with each other for five minutes (Jacome et al., in press); thus, the social stimulus mouse will make significantly more discrete episodes of social approach toward the Balb/c test mouse than toward the outbred Swiss Webster comparator strain during this period of free interaction. The Balb/c mouse also demonstrates less anogenital sniffing and "mounting (i.e., placing forepaws on the stimulus mouse)" of the stimulus mouse, compared with the outbred Swiss Webster comparator strain. Another interesting behavioral feature of the Balb/c mouse is the dissociation between impaired sociability and intensity of spontaneous self-grooming behavior and other stereotypic behaviors (Jacome et al., in press). Thus, relative to the outbred Swiss Webster comparator strain, the Balb/c mouse spends less time engaged in spontaneous self-grooming and other stereotypic behaviors during the five-minute period of free interaction between test (i.e., Balb/c or Swiss Webster) and social stimulus (i.e., ICR strain) mice. This latter finding was unexpected in view of the fact that stereotypies (e.g., hand-flapping) are frequently observed among persons with autism spectrum disorders; self-grooming, rearing, and wall-climbing are examples of stereotypic behaviors measured in mice. 
Because the Balb/c mouse is behaviorally-hypersensitive to MK-801, suggesting an altered endogenous tone of NMDA receptor-mediated neurotransmission, we wondered if pharmacological interventions targeting the NMDA receptor would affect its social behavior in a standard paradigm. The initial study examined D-cycloserine $(320 \mathrm{mg} / \mathrm{kg}$, intraperitoneally), a partial glycine agonist. This study confirmed that 8-week old male Balb/c mice made significantly fewer discrete episodes of operationally-defined social approach toward a 4-week old male ICR social stimulus mouse when the two were allowed to interact freely with each other, compared with the same measure of social approach of 8week old male Swiss Webster mice in the same paradigm (Deutsch et al., 2011). Remarkably, however, the number of discrete episodes of social approach toward the social stimulus mouse made by Balb/c mice treated with D-cycloserine $(320 \mathrm{mg} / \mathrm{kg}$, intraperitoneally) did not differ from the vehicle-treated Swiss Webster mice. Moreover, the locomotor activity of D-cycloserine-treated Balb/c mice in the presence of both an enclosed and freely-behaving social stimulus mouse did not differ from the locomotor activity of vehicle-treated Swiss Webster mice under the same conditions. Thus, D-cycloserine attenuated the severity of several quantitative measures of the Balb/c mouse's deficit of sociability, restoring these measures to levels observed in vehicle-treated Swiss Webster mice, the outbred comparator strain (Deutsch et al., 2011). In further studies with D-cycloserine designed to explore possible mechanisms of its effect(s) and an explanation for the Balb/c mouse's deficit of sociability, we showed that Balb/c mice did not differ from Swiss Webster mice in terms of time spent exploring an inanimate object, whereas, as expected, Balb/c mice spent significantly less time exploring an enclosed social stimulus mouse (Jacome et al., 2011a). Moreover, the Balb/c mouse's deficit in time spent in social exploration was not an epiphenomenon of a greater basal level of "anxiety" because Balb/c mice did not spend less time in the open arms of an elevated plus maze than the Swiss Webster comparator strain. Also, D-cycloserine (320 mg/ kg, intraperitoneally) significantly increased the number of open-arm entries of both strains of mice, suggesting that it causes a general increase in exploratory activity that was not strain-specific. However, there was something unique about a social stimulus mouse's ability to suppress the locomotor activity of the Balb/c strain and D-cycloserine's strain-selective ability to increase social exploration in the genetically-inbred, sociability-impaired Balb/c strain; there was no similar D-cycloserineinduced increase of social exploration in the Swiss Webster strain (Jacome et al., 2011a).

In addition to D-cycloserine, our laboratory showed that D-serine $(560 \mathrm{mg} / \mathrm{kg}$, intraperitoneally), a full glycine agonist, attenuated the severity of deficits of sociability in the Balb/c strain (Jacome et al., 2011b). The ability of D-serine to do this serves as an important "proof of concept" that targeted NMDA receptor agonist interventions can improve sociability in a genetic mouse model with presumed disturbance of endogenous NMDA receptor-mediated neurotransmission. Additionally, D-serine, like D-cycloserine, improved sociability in young adult 8-week old mice that serve as a genetic mouse model for at least some dimensions of autism spectrum disorders; these data encourage development of medication interventions in a neurodevelopmental disorder. Specifically, Dserine increased the amount of time Balb/c mice spent both in the compartment containing the enclosed social stimulus mouse and the amount of time Balb/c mice spent actually exploring and sniffing the enclosed social stimulus mouse (Jacome et al., 2011b). Importantly, the Swiss Webster comparator strain spent significantly more time in the compartment containing the enclosed social stimulus mouse and spent significantly more time exploring and sniffing this enclosed mouse even in the vehicle-treated condition. 
However, as noted earlier, there are serious concerns with the development of a full agonist for the treatment of a chronic neuropsychiatric/neurodevelopmental disorder, such as an autism spectrum disorder. Specifically, a full agonist may have diminished efficacy/effectiveness over time because of agonist-induced desensitization, and a full agonist may cause excitotoxicity. Thus, D-cycloserine, a partial glycine agonist, may be expected to better retain efficacy/effectiveness upon chronic administration, while not being associated with increased risk for excitotoxicity; however, this is an empirical question that we will pursue in future studies.

As discussed, the mGluR5 receptor is functionally coupled to NMDA receptors; mGluR5 is a GPCR that is coupled to a Gq protein, whose biochemical effector is phospholipase $\mathrm{C} \beta$ (Borowicz et al., 2009; Niswender \& Conn, 2010). Importantly, in addition to stimulating phosphorylation of a serine residue in the intracytoplasmic domain of the NR1 subunit of the NMDA receptor and, thereby, increasing the sensitivity of the NMDA receptor to stimulation by glutamate and glycine, mGluR5 receptors also play an important role in regulating translation of proteins in the basilar dendrites of pyramidal cell neurons. Stimulation of the mGluR5 receptor promotes protein synthesis (Bear et al., 2004; Dölen et al., 2010); thus, mGluR5's promotion of protein synthesis is unopposed in persons with Fragile $X$ Syndrome, who are deficient in expression of the 'fragile $X$ mental retardation protein." A major role of the 'fragile X mental retardation protein' is to dampen protein synthesis in basilar dendrites through its interaction and interference with the function of polyribosomes (Bear et al., 2004; Dölen et al., 2010). Because an unbalanced increase in protein synthesis in basilar dendrites is thought to contribute to the pathophysiological disturbance of Fragile $X$ Syndrome, which has a high co-morbid prevalence of autism spectrum disorders, mGluR5 antagonism is emerging as a promising therapeutic strategy for this X-linked disorder.

The high comorbid prevalence of autism spectrum disorders among persons with Fragile $X$ Syndrome and the beneficial effect of mGluR5 antagonism in mouse models of Fragile $X$ Syndrome stimulated exploration of mGluR5 antagonism in another genetically-inbred mouse model of autism spectrum disorders (Silverman et al., 2010). Specifically, in a recent study, 2methyl-6-(phenylethynyl)-pyridine (MPEP), an mGluR5 receptor antagonist that is centrally available after peripheral administration, was shown to attenuate the severity of self-grooming in BTBR T + tfj (BTBR) mice, an inbred genetic mouse model of autism spectrum disorders; the intervention did not affect locomotor activity nor did it have an effect on the impaired sociability of this mouse strain (Silverman et al., 2010). Repetitive self-grooming is thought to reflect and model the symptom domain of "restricted repetitive and stereotyped patterns of behavior," which is an important dimension of psychopathology in autism spectrum disorders. (In fact the text revision of the fourth edition of the American Psychiatric Association's Diagnostic and Statistical Manual requires that definitional criteria for at least one item/symptom in this domain must be fulfilled in order to assign a diagnosis of "autistic disorder.") Importantly, the BTBR mouse strain, in contrast to the Balb/c mouse strain, showed more intense repetitive self-grooming behavior than the comparator C57BL/6J mouse strain used in these studies (Silverman et al., 2010), the latter is a mouse strain characterized as displaying "high-sociability." Because of the interest in MPEP as a pharmacological intervention for a significant domain of autism psychopathology (i.e., "restricted repetitive and stereotyped patterns of behavior"), our group decided to study the effect of MPEP $(30 \mathrm{mg} / \mathrm{kg}$, intraperitoneally-administered) on measures of sociability and stereotypies, including spontaneous self-grooming behavior, in test mice (i.e., Balb/c or Swiss Webster) while they 
interacted freely with stimulus mice (i.e., ICR mice); as noted MPEP is a relatively selective, potent $\left(\mathrm{IC}_{50}\right.$ value in the $30 \mathrm{nM}$ range), blood-brain barrier permeable noncompetitive mGluR5 antagonist (Gasparini et al., 1999). Because the baseline level of stereotypies in the Balb/c strain is low, it is hard to detect drug effects on stereotypy outcome measures in this strain. However, the findings in the comparator Swiss Webster strain on reduction of stereotypies and worsening of some measures of sociability by MPEP underscore the need for caution in the development of mGluR5 antagonists as medications for the treatment of ASDs. Importantly, MPEP significantly reduced stereotypies in the Swiss Webster comparator strain, suggesting that this domain of autism spectrum disorder psychopathology may be uncoupled from sociability, reflecting, perhaps, their differences in underlying circuitry. MPEP also had socially disruptive effects in both strains (Burket et al., submitted manuscript), as well as some positive effects on sociability, thus, while MPEP attenuated stereotypies that appeared spontaneously during social interaction in both strains, it also had complex and inconsistent effects, including worsening, on sociability.

Given the role of the NMDA receptor for the display of normal sociability, these data add a cautionary note with respect to the pursuit of an mGluR5 antagonist medication strategy in autism. As a result of "cross-talk" between mGluR5 and NMDA receptors, stimulation of mGluR5 can promote phosphorylation of a serine residue in the intracytoplasmic domain of the NR1 subunit, which should result in "activation" of the NMDA receptor and its greater sensitivity to channel opening in response to glutamate and glycine (Mario \& Conn et al., 2002). Moreover, MPEP is believed to exert its mGluR5 inhibitory properties by binding or interacting with a site in the seven transmembranous hydrophobic domain that is also the site of action of several positive allosteric modulators (PAMs) of mGluR5 (Conn et al., 2009b). Given the fact administration of glutamate "agonists" lack specificity and bind to a diverse variety of metabotropic and ionotropic glutamate receptors, in addition to their potential for excitotoxicity, interest has shifted to development of positive and negative allosteric modulators to selectively increase and decrease, respectively, signal transduction by metabotropic glutamate receptors (Conn et al., 2009a). Unlike the agonist recognition sites themselves that show conservation of their electronic architecture for recognition of glutamate and its analogues, allosteric modulatory sites show diversity of structure among glutamate receptors, affording "selectivity (i.e., only selected metabotropic and ionotropic glutamate receptors will be targeted)." Also, allosteric modulators are only effective in the presence of the endogenous agonist (Conn et al., 2009a); thus, they will be effective only at the precise synapse that glutamate (and glycine) is released, preserving the temporal and spatial constraints that are so critical to efficient, as opposed to "leaky," synaptic transmission. We and others are beginning to explore the potential therapeutic role of positive allosteric modulators of mGluR5 as a medication strategy to address presumptive NMDA receptor hypofunction in autism spectrum disorders.

\section{Future directions and translational applications}

Targeting the NMDA receptor and circuits containing and utilizing the NMDA receptor for synaptic transmission remain a promising area of future investigations. Very importantly, preclinical studies must also include chronic dosing conditions and the demonstration of sustained efficacy. Viable strategies should include manipulations both upstream and downstream of the presumed location of NMDA receptors on the surface of GABA 
inhibitory neurons, especially the "fast-spiking (FS)," parvalbumin-containing GABA neurons that regulate assemblies of pyramidal outflow neurons and underlie gamma oscillations (Benes et al., 2010). (In addition to NMDA receptors, specific kainate receptors, another type of ionotropic glutamate receptor, on the surface of GABA inhibitory interneurons [e.g., GluR6-containing kainate receptors] may also contribute to the generation and regulation of important oscillatory rhythms in brain.)

Thus, future studies can explore the acute and chronic consequences of both reducing (e.g., stimulating glutamate reuptake with guanosine) and increasing (e.g., via specific glutamate reuptake inhibitors) the concentration of glutamate within the synaptic cleft.

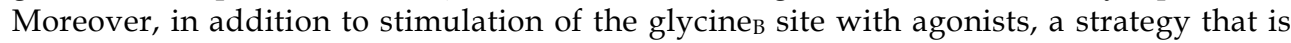
supported by the results of the D-serine and D-cycloserine experiments discussed in this review, interference with presynaptic reuptake of glycine via the use of glycine-1 transporter inhibitors (e.g., sarcosine and its derivatives) is another approach. We have reviewed the rationale underlying the use of PAMs of mGluR5 above; this strategy could conceivably be expanded or complemented by strategies that inhibit the activities of specific "regulators of G protein signaling (e.g., RGS4)." "Regulators of G protein signaling" promote the activities of GTPase and, thereby, dampen production of the soluble intracellular secondary messengers, whose synthesis is activated by glutamate's stimulation of metabotrobic glutamate receptors. The genetically-inbred, sociabilityimpaired Balb/c mouse may also be used to study the effects of neurosteroids on sociability; neurosteroids act at the level of cell surface ionotropic receptors (e.g., GABA and NMDA receptors) to influence ion flux and membrane potential in subtle ways. Other strategies could include downstream manipulations; for example, if the GABAergic neurons containing NMDA receptors on their surface are dysfunctional, selective benzodiazepine agonist interventions could be considered (e.g., agonists that target $\mathrm{GABA}_{\mathrm{A}}$ receptors containing a 3 or a5 subunits) that would promote GABAergic transmission downstream, via bypassing or compensating for the "defective" GABA inhibitory neuron. Additionally, if release of GABA is diminished, downstream glutamatergic projections could be relieved of critical GABAergic inhibition, which might be addressed by selective glutamate receptor antagonists that would block or attenuate the consequences of excessive downstream release of glutamate. Clearly, this is a highly speculative discussion, but the availability of genetic mouse models of autism spectrum disorders in general, and impaired sociability in particular, tested in a standard paradigm to assess sociability encourage and make this type of systematic examination possible.

There are provocative data suggesting that D-cycloserine improved social withdrawal in a small sample of 10 children with autism (Posey et al., 2004). Clearly, the therapeutic effect of a medication in this neurodevelopmental disorder is expected to be small and clinical trials must minimize "noise" associated with subject differences that may obscure a positive effect of treatment. Nonetheless, a medication that targets domains of sociability and cognition in the context of a well-designed interdisciplinary, individualized treatment plan that includes components of special education, speech and language therapy, occupational therapy and vocational assessment and training may contribute to significantly improved quality of life for many persons suffering with autism spectrum disorders. An outline for an integrated therapeutic strategy to address the sociability deficit of persons with autism spectrum disorders is shown in Table 3; clearly, medications will be only one component, albeit an important one, of a comprehensive individualized, interdisciplinary, multimodal treatment plan. 
The NMDA receptor contributes to regulation of central inhibitory tone by influencing the firing of GABA inhibitory neurons. In addition to NMDA receptors, other receptors on the surface of the GABA inhibitory neuron may represent promising pharmacotherapeutic targets. In particular, the $\alpha_{7}$ subunit-containing nicotinic acetylcholine receptor $\left(\alpha_{7} \mathrm{nAChR}\right)$, like the NMDA receptor, is a ligand-gated primarily calcium ion channel receptor on the surface of GABA inhibitory neurons that is emerging as a promising target. Recent efficient, high-throughput strategies for interrogating the human genome in large populations of referred persons with developmental disabilities showed that microdeletions of 15q13.3 (i.e., copy number variants), the locus of the $a_{7} \mathrm{nAChR}$, are associated with presentations of ASDs, which lend genetic support for the development of medications that target the a7nAChR. Hopefully, medication development strategies that exploit these promising genetic leads in appropriate mouse models will lead to improved treatments and outcomes for ASDs (Deutsch et al., submitted for publication).



\section{References}

American Psychiatric Association. (2000). Diagnostic and statistical manual of mental disorders, (4th ed., text revision), American Psychiatric Association, Washington, DC.

Ashwin, C., Baron-Cohen, S., Wheelwright, S., O'Riordan, M., Bullmore, E.T. (2007). Differential activation of the amygdala and the 'social brain' during fearful faceprocessing in Asperger Syndrome. Neuropsychologia, 45, 1, 2-14.

Bear, M.F., Huber, K.M., Warren, S.T. (2004). The mGluR theory of fragile X mental retardation. Trends in Neuroscience, 27, 370-7.

Benes, F.M. (2010). Amygdalocortical circuitry in schizophrenia: from circuits to molecules. Neuropsychopharmacology, 35, 1, 239-57. 
Billingslea, E.N., Mastropaolo, J., Rosse, R.B., Bellack, A.S., Deutsch, S.I. (2003). Interaction of stress and strain on glutamatergic neurotransmission: relevance to schizophrenia. Pharmacology, Biochemistry, \& Behavior, 74, 351-6.

Blundell, J., Blaiss, C.A., Etherton, M.R., Espinosa, F., Tabuchi, K., Walz, C., Bolliger, M.F., Südhof, T.C., Powell, C.M. (2010). Neuroligin-1 deletion results in impaired spatial memory and increased repetitive behavior. Journal of Neuroscience, 30, 2115-29.

Borowicz, K.K., Łuszczki, J.J., Czuczwar, S.J. (2009). 2-Methyl-6-phenylethynyl-pyridine (MPEP), a non-competitive mGluR5 antagonist, differentially affects the anticonvulsant activity of four conventional antiepileptic drugs against amygdalakindled seizures in rats. Pharmacological Reports, 61, 621-30.

Bremer, A., Giacobini, M., Eriksson, M., Gustavsson, P., Nordin, V., Fernell, E., Gillberg, C., Nordgren, A., Uppströmer, A., Anderlid, B.M., Nordenskjöld, M., Schoumans, J. (2011). Copy number variation characteristics in subpopulations of patients with autism spectrum disorders. American Journal of Medical Genetics Part B: Neuropsychiatric Genetics, 156, 2, 115-24.

Brodkin, E.S. (2007). BALB/c mice: low sociability and other phenotypes that may be relevant to autism. Behavioral Brain Research, 176, 53-65.

Burket, J.A., Cannon, W.R., Jacome, L.F., \& Deutsch, S.I. (2010a). MK-801, a Noncompetitive NMDA Receptor Antagonist, Elicited Circling Behavior in the Genetically-Inbred Balb/c Mouse Strain. Brain Research Bulletin, 83, 337-9.

Burket, J.A., Herndon, A.L., \& Deutsch, S.I. (2010b). Locomotor activity of the geneticallyinbred mouse strain is suppressed by a socially-salient stimulus. Brain Research Bulletin, 83, 255-256.

Burket, J.A., Mastropaolo, J., Rosse, R.B., Deutsch, S.I. (2009). Genetically inbred Balb/C mice are more sensitive to an effect of flurazepam and more resistant to an effect of stress than a genetically outbred mouse strain. Epilepsy \& Behavior, 16, 3, 415-7.

Burket, J.A., Herndon, A.L., Winebarger, E.E., Jacome, L.F., Deutsch, S.I. (n.d.). Divergent Effects of mGluR5 Antagonism on Sociability and Stereotypic Behaviors in Mice: Possible Implications for the Pharmacotherapy of Autism Spectrum Disorders, submitted manuscript.

Chen, L. \& Huang, L.Y. (1992). Protein kinase C reduces Mg2+ block of NMDA-receptor channels as a mechanism of modulation. Nature, 356, 521-3.

Conn, P.J., Christopoulos, A., Lindsley, C.W. (2009a). Allosteric modulators of GPCRs: a novel approach for the treatment of CNS disorders. National Reviews Drug Discovery, 8, 1, 41-54.

Conn, P.J., Lindsley, C.W., Jones, C.K. (2009b). Activation of metabotropic glutamate receptors as a novel approach for the treatment of schizophrenia. Trends in Pharmacological Sciences, 30, 1, 25-31.

Crawley, J.N. (2004). Designing mouse behavioral tasks relevant to autistic-like behaviors. Mental Retardation and Developmental Disabilities Research Reviews, 10, 248-258.

Crawley, J.N. (2007). Mouse Behavioral Assays Relevant to the Symptoms of Autism. Brain Pathology, 17, 448-59.

Darrah, J.M., Stefani, M.R., Moghaddam, B. (2008). Interaction of N-methyl-D-aspartate and group 5 metabotropic glutamate receptors on behavioral flexibility using a novel operant set-shift paradigm. Behavioral Pharmacology, 19, 225-34. 
Deutsch, S.I., Burket, J.A., Jacome, L.F., Cannon, W.R., \& Herndon, A.L. (2011). DCycloserine Improves the Impaired Sociability of the Balb/c Mouse. Brain Research Bulletin, 84, 8-11.

Deutsch, S.I., Mastropaolo, J., Hitri, A. (1992). GABA-active steroids: endogenous modulators of GABA-gated chloride ion conductance. Clinical Neuropharmacology, $15,5,352-64$.

Deutsch, S.I., Mastropaolo, J., Powell, D.G., Rosse, R.B., Bachus, S.E. (1998). Inbred mouse strains differ in their sensitivity to an antiseizure effect of MK-801. Clinical Neuropharmacology, 21, 255-7.

Deutsch, S.I., Rosse, R.B., Huntzinger, J.A., Novitzki, M.R., Mastropaolo, J. (1990). Profound stress-induced alterations in flurazepam's antiseizure efficacy can be attenuated. Brain Research, 520, 1-2, 272-6.

Deutsch, S.I., Rosse, R.B., Paul, S.M., Riggs, R.L., \& Mastropaolo, J. (1997). Inbred mouse strains differ in sensitivity to popping elicited by MK-801. Pharmacology, Biochemistry \& Behavior, 57, 315-317.

Deutsch, S.I., Rosse, R.B., Schwartz, B.L., Mastropaolo, J., Burket, J.A., Weizman, A. (2010). Regulation of intermittent oscillatory activity of pyramidal cell neurons by GABA inhibitory interneurons is impaired in schizophrenia: rationale for pharmacotherapeutic GABAergic interventions. Israel Journal of Psychiatry \& Related Sciences, 47, 1, 17-26.

Deutsch, S.I., Urbano, M.R., Burket, J.A., Herndon, A.L., Winebarger, E.E. (n.d). Pharmacotherapeutic Implications of the Association between Genomic Instability at Chromosome 15q13.3 and Autism Spectrum Disorders, submitted manuscript.

Dölen, G., Carpenter, R.L., Ocain, T.D., Bear, M.F. (2010). Mechanism-based approaches to treating fragile X. Pharmacology \& Therapeutics, 127, 1, 78-93.

Duncan, G.E., Moy, S.S., Perez, A., Eddy, D.M., Zinzow, W.M., Lieberman, J.A., Snouwaert, J.N., Koller, B.H. (2004). Deficits in sensorimotor gating and tests of social behavior in a genetic model of reduced NMDA receptor function. Behavioural Brain Research, 153, 2, 507-19.

Gasparini, F., Lingenhöhl, K., Stoehr, N., Flor, P.J., Heinrich, M., Vranesic, I., et al. (1999). 2Methyl-6-(phenylethynyl)-pyridine (MPEP), a potent, selective and systemically active mGlu5 receptor antagonist. Neuropharmacology, 38, 1493-503.

Gregory, K.J., Dong, E.N., Meiler, J., Conn, P.J. (2011). Allosteric modulation of metabotropic glutamate receptors: structural insights and therapeutic potential. Neuropharmacology, 60, 1, 66-81.

Halene, T.B., Ehrlichman, R.S., Liang, Y., Christian, E.P., Jonak, G.J., Gur, T.L., Blendy, J.A., Dow, H.C., Brodkin, E.S., Schneider, F., Gur, R.C., Siegel, S.J. (2009). Assessment of NMDA receptor NR1 subunit hypofunction in mice as a model for schizophrenia. Genes, Brain E Behavior, 8, 661-75.

Jacome, L.F., Burket, J.A., Herndon, A.L., Deutsch, S.I. (2011a). D-cycloserine Enhances Social Exploration in the Balb/c Mouse. Brain Research Bulletin, 85,3-4, 141-4.

Jacome, L.F., Burket, J.A., Herndon, A.L., Deutsch, S.I. (n.d.) Genetically-Inbred Balb/c Mice Differ from Outbred Swiss Webster Mice on Discrete Measures of Sociability: Relevance to a Genetic Mouse Model of Autism Spectrum Disorders, in press. 
Jacome, L.F., Burket, J.A., Herndon, A.L., Cannon, W.R., \& Deutsch, S.I. (2011b). D-serine Improves Dimensions of the Sociability Deficit of the Genetically-Inbred Balb/c Mouse Strain. Brain Research Bulletin, 84, 12-6.

Kew, J.N. \& Kemp, J.A. (2005). Ionotropic and metabotropic glutamate receptor structure and pharmacology. Psychopharmacology, 179, 1, 4-29.

Kleinhans, N.M., Richards, T., Johnson, L.C., Weaver, K.E., Greenson, J., Dawson, G., Aylward, E. (2011). fMRI evidence of neural abnormalities in the subcortical face processing system in ASD. Neuroimage, 54, 1, 697-704.

Labrie, V., Lipina, T., Roder, J.C. (2008). Mice with reduced NMDA receptor glycine affinity model some of the negative and cognitive symptoms of schizophrenia. Psychopharmacology, 200, 2, 217-230.

Long, K.D., Mastropaolo, J., Rosse, R.B., Manaye, K.F., Deutsch, S.I. (2006). Modulatory effects of d-serine and sarcosine on NMDA receptor-mediated neurotransmission are apparent after stress in the genetically inbred BALB/c mouse strain. Brain Research Bulletin, 69, 6, 626-30.

Marino, M.J. \& Conn, P.J. (2002). Direct and indirect modulation of the N-methyl Daspartate receptor. Current Drug Targets - CNS \& Neurological Disorders, 1, 1, 1-16.

McAllister, K.H. (1994). D-Cycloserine enhances social behavior in individually-housed mice in the resident-intruder test. Psychopharmacology, 116, 317-325.

Millan, M.J. (2005). N-Methyl-D-aspartate receptors as a target for improved antipsychotic agents: novel insights and clinical perspectives. Psychopharmacology, 179, 1, 30-53.

Moy, S.S., Nadler, J.J., Young, N.B., Perez, A., Holloway, L.P., Barbaro, R.P., et al. (2007). Mouse behavioral tasks relevant to autism: phenotypes of 10 inbred strains. Behavioral Brain Research, 176, 4-20.

Niswender, C.M. \& Conn, P.J. (2010). Metabotropic glutamate receptors: physiology, pharmacology, and disease. Annual Review of Pharmacology and Toxicology, 50, 295322.

Noterdaeme, M., Wriedt, E., Höhne, C. (2010). Asperger's syndrome and high-functioning autism: language, motor and cognitive profiles. European Child $\mathcal{E}$ Adolescent Psychiatry, 19, 6, 475-81.

Perera, P.Y., Lichy, J.H., Mastropaolo, J., Rosse, R.B., Deutsch, S.I. (2008). Expression of NR1, NR2A and NR2B NMDA receptor subunits is not altered in the genetically-inbred Balb/c mouse strain with heightened behavioral sensitivity to MK-801, a noncompetitive NMDA receptor antagonist. European Journal of Neuropsychopharmacology, 18, 814-9.

Posey, D.J., Kem, D.L., Swiezy, N.B., Sweeten, T.L., Wiegand, R.E., McDougle, C.J. (2004). A pilot study of D-cycloserine in subjects with autistic disorder. American Journal of Psychiatry, 161, 2115-7.

Sankoorikal, G.M., Kaercher, K.A., Boon, C.J., Lee, J.K., \& Brodkin, E.S. (2006). A mouse model system for genetic analysis of sociability: C57BL/6J versus BALB/cJ inbred mouse strains. Biological Psychiatry, 59, 415-23.

Silverman, J.L., Tolu, S.S., Barkan, C.L., Crawley, J.N. (2010). Repetitive self-grooming behavior in the BTBR mouse model of autism is blocked by the mGluR5 antagonist MPEP. Neuropsychopharmacology, 35, 4, 976-89.

Stephenson, F.A. (2006). Structure and trafficking of NMDA and GABAA receptors. Biochemical Society Transactions, 34, Pt 5, 877-81. 
Takagi, N., Besshoh, S., Morita, H., Terao, M., Takeo, S., Tanonaka, K. (2010). Metabotropic glutamate mGlu5 receptor-mediated serine phosphorylation of NMDA receptor subunit NR1 in hippocampal CA1 region after transient global ischemia in rats. European Journal of Pharmacology, 644, 96-100.

Toro, R., Konyukh, M., Delorme, R., Leblond, C., Chaste, P., Fauchereau, F., Coleman, M., Leboyer, M., Gillberg, C., Bourgeron, T. (2010). Key role for gene dosage and synaptic homeostasis in autism spectrum disorders. Trends in Genetics, 26, 8, 363-72. 


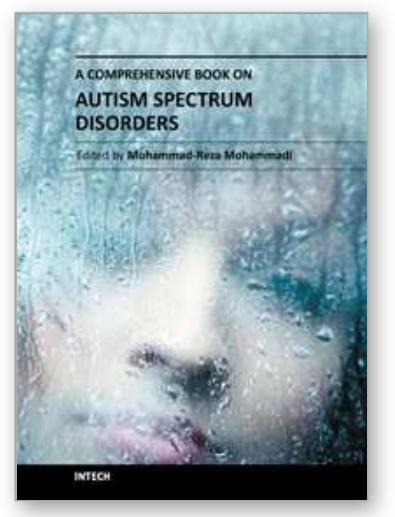

\section{A Comprehensive Book on Autism Spectrum Disorders}

Edited by Dr. Mohammad-Reza Mohammadi

ISBN 978-953-307-494-8

Hard cover, 478 pages

Publisher InTech

Published online 15, September, 2011

Published in print edition September, 2011

The aim of the book is to serve for clinical, practical, basic and scholarly practices. In twentyfive chapters it covers the most important topics related to Autism Spectrum Disorders in the efficient way and aims to be useful for health professionals in training or clinicians seeking an update. Different people with autism can have very different symptoms.Â Autism is considered to be a â€œspectrumâ€ disorder, a group of disorders with similar features. Some people may experience merely mild disturbances, while the others have very serious symptoms. This book is aimed to be used as a textbook for child and adolescent psychiatry fellowship training and will serve as a reference for practicing psychologists, child and adolescent psychiatrists, general psychiatrists, pediatricians, child neurologists, nurses, social workers and family physicians. A free access to the full-text electronic version of the book via Intech reading platform at http://www.intechweb.org is a great bonus.

\section{How to reference}

In order to correctly reference this scholarly work, feel free to copy and paste the following:

Stephen I. Deutsch, Jessica A. Burket, Maria R. Urbano, Amy L. Herndon and Erin E. Winebarger (2011). Impaired Sociability of the Balb/c Mouse, an Animal Model of Autism Spectrum Disorders, is Attenuated by NMDA Receptor Agonist Interventions: Clinical Implications, A Comprehensive Book on Autism Spectrum Disorders, Dr. Mohammad-Reza Mohammadi (Ed.), ISBN: 978-953-307-494-8, InTech, Available from: http://www.intechopen.com/books/a-comprehensive-book-on-autism-spectrum-disorders/impaired-sociabilityof-the-balb-c-mouse-an-animal-model-of-autism-spectrum-disorders-is-attenuated-

\section{INTECH}

open science | open minds

\section{InTech Europe}

University Campus STeP Ri

Slavka Krautzeka 83/A

51000 Rijeka, Croatia

Phone: +385 (51) 770447

Fax: +385 (51) 686166

www.intechopen.com

\section{InTech China}

Unit 405, Office Block, Hotel Equatorial Shanghai

No.65, Yan An Road (West), Shanghai, 200040, China 中国上海市延安西路65号上海国际贵都大饭店办公楼 405 单元

Phone: +86-21-62489820

Fax: +86-21-62489821 
(C) 2011 The Author(s). Licensee IntechOpen. This chapter is distributed under the terms of the Creative Commons Attribution-NonCommercialShareAlike-3.0 License, which permits use, distribution and reproduction for non-commercial purposes, provided the original is properly cited and derivative works building on this content are distributed under the same license. 